\title{
Some Recent Results in Finitely Additive White Noise Theory
}

\author{
ARUNABHA B A C H I \\ Department of Applied Mathematics, University of Twente, P.O. Box 217, \\ 7500 AE Enschede, The Netherlands \\ and \\ RAVI MAZUMDAR \\ INRS-Telecommunications, 3, Place du Commerce, Verdun, PQ, H3E 1 H6 Canada
}

(Received: 13 July 1993)

\begin{abstract}
We present a short survey of some very recent results on the finitely additive white noise theory. We discuss the Markov property of the solution of a stochastic differential equation driven directly by a white noise, study the Radon-Nikodym derivative of the measure induced by nonlinear transformation on a Hilbert space with respect to the canonical Gauss measure thereon and obtain a representation for nonlinear filter maps.
\end{abstract}

Mathematics Subject Classifications (1991). 60H10, 93E11.

Key words. Finitely additive white noise, Markov property, Radon-Nikodym derivative, nonlinear filter.

\section{Introduction}

Nonlinear stochastic differential systems are usually modelled by using the theory of stochastic differential equations as developed by Itô [13]. There is a rich theory of nonlinear estimation, likelihood ratio and stochastic control based on this theory $[9,14]$. The resulting equations hold, as is expected in probability theory, almost surely in the space of continuous functions. This causes a serious difficulty when one handles real data. The reason is that, in this approach, the data is modelled in the 'integral form' and is nowhere differentiable because of the pathological sample-path property of a Brownian motion. In practice, however, the data is always absolutely continuous and, in Wiener space, the set of absolutely continuous functions has measure zero! Thus, results obtained by this approach are not directly applicable to real data.

One way to circumvent this difficulty, which is originally due to Balakrishnan [4], is to try to model the observation process directly with a white noise input. Although modeling white noise directly is intuitively appealing, it brings a host of mathematical complications. This is because, in this theory, one has to work with 
finitely additive measures on an appropriate Hilbert space and these measures cannot be extended to be countably additive on that space. Kallianpur and Karandikar [15] developed a comprehensive theory of nonlinear estimation in this framework. The remarkable advantage of this theory is that, once the mathematical difficulties are resolved, the results obtained are always in the form where real data can be directly used. In technical terms we say that the results are always in the robust form.

Until recently one drawback of this finitely additive white noise theory was that the state process had to be modelled as the solution of an Itô stochastic differential equation, while the observation process was modelled directly with an additive white noise term. Recent works on stochastic differential equations with a 'finitely additive' white noise as the input helps one in avoiding this asymmetry for some cases. The first difficulty one encounters is to show that the solution of such a stochastic differential equation is a Markov process. Once this issue is successfully resolved, a host of new results are obtained. This will be the subject matter of this paper.

The organization of the paper is as follows. In Section 2, we present, in short, the necessary mathematical preliminaries. In Section 3, we discuss the Markov property mentioned above and give the robust solution of the nonlinear estimation problem with correlated state and observation noises. In Section 4, we study Radon-Nikodym derivatives of finitely additive measures induced by nonlinear transformations. This is the white noise analog of the celebrated work of Cameron and Martin [7] on nonlinear transformation of the Wiener measure. We use this result to obtain the Wong-Zakai correction term [24]. In Section 5 we obtain a representation for nonlinear filter maps and point out its connection with finite-dimensional filters. In Section 6 , we mention some open problems and present some conclusions.

\section{Mathematical Preliminaries}

We work throughout with the separable Hilbert space $H=L_{2}\left([0, T] ; \mathbb{R}^{n}\right)$ as the sample space. We denote the inner product in $H$ by $[\cdot, \cdot]$ and norm by $\|\cdot\|$. Let $\mathcal{P}$ be the set of all orthogonal projections on $H$ with finite-dimensional range. We introduce a partial ordering on $\mathcal{P}$ by defining $P_{1} \leqslant P_{2}$ if (Range $\left.P_{1}\right) \subseteq$ (Range $P_{2}$ ), for $P_{1}, P_{2} \in \mathcal{P}$.

For $P \in \mathcal{P}$, let

$$
\left.\mathcal{C}_{P}=\left\{P^{-1} B \mid B \text { Borel in (Range } P\right)\right\}
$$

and define

$$
\mathcal{C}=\bigcup_{P \in \mathcal{P}} \mathcal{C}_{P}
$$

Sets in $\mathcal{C}$ are called cylinder sets in $H$. Each $\mathcal{C}_{P}$ for fixed $P \in \mathcal{P}$, is a $\sigma$-algebra, but $\mathcal{C}$ is only an algebra. 
A cylinder measure $\lambda$ on $H$ is a finitely additive measure on $(H, \mathcal{C})$ such that for all $P \in \mathcal{P}$, the restriction $\lambda_{P}$ of $\lambda$ to $\mathcal{C}_{P}$ is countably additive. If $\lambda(H)=1$, we say that $\lambda$ is a cylinder probability. The canonical Gauss measure $\mu$ on $(H, \mathcal{C})$ is a cylinder probability on $H$ such that, for all $h \in H$,

$$
\int_{H} \exp \left\{i\left[h, h_{1}\right]\right\} \mathrm{d} \mu\left(h_{1}\right)=\exp \left\{-\frac{1}{2}\|h\|^{2}\right\} \text {. }
$$

When we do not specifically mention the cylinder probability, we mean it to be the canonical Gauss measure $\mu$.

Let $\mathcal{B}$ denote the Borel $\sigma$-algebra of $H$; it is the smallest $\sigma$-algebra containing $\mathcal{C}$. Since $\mu$ cannot be extended to be countably additive on $\mathcal{B}$, most results of measure theory cannot be directly used when we work with $\mu$. For example, let $f: H \rightarrow \mathbb{R}$ be a Borel measurable map. This does not qualify $f$ to be a random variable, since the inverse images $f^{-1}(\widetilde{B}), \widetilde{B}$ any Borel set in $\mathbb{R}$, are not necessarily in $\mathcal{C}$ and probabilities can not be assigned to these 'events'.

DEFINITION 2.1. A function $f: H \rightarrow \mathbb{R}$ belongs to $\mathcal{L}(H, \mathcal{C}, \lambda)$ if $f$ is Borel measurable and the net $\left\{f_{P} \mid P \in \mathcal{P}\right\}$ of functions defined by

$$
f_{P}(h)=f(P h)
$$

is Cauchy in $\lambda$-measure. Elements of $\mathcal{L}(H, \mathcal{C}, \lambda)$ will be called $\lambda$-random variables when $\lambda$ is a cylinder probability. We call $\mu$-random variables simply random variables. It is easy to extend the definition to the case when $f: H \rightarrow \mathbb{R}^{n}$, or what will often be used in the sequel, when $f: H \rightarrow H^{\prime}$ with $H^{\prime}$ also a separable Hilbert space. We denote the corresponding classes of $\lambda$-random variables by $\mathcal{L}\left(H, \mathcal{C}, \lambda ; \mathbb{R}^{n}\right)$ or $\mathcal{L}\left(H, \mathcal{C}, \lambda ; H^{\prime}\right)$, respectively. It is also straightforward to define $\mathcal{L}(H, \mathcal{C}, \lambda ; S)$, where $S$ is a separable metric space.

For $f \in \mathcal{L}\left(H, \mathcal{C}, \lambda ; H^{\prime}\right)$, it can be shown that the net of measures $\left\{\lambda \circ f_{P}^{-1}, P \in \mathcal{P}\right\}$ converges to a countably additive measure on $H^{\prime}$. The limit is called the measure induced by $f$ under $\lambda$, and is denoted by $\lambda \circ f^{-1}$. By the class $\mathcal{L}_{1}(H, \mathcal{C}, \lambda)$ of integrable random variables we mean the elements $f \in \mathcal{L}(H, \mathcal{C}, \lambda)$ for which

$$
\int_{\mathbb{I}} x \mathrm{~d}\left(\lambda \circ f^{-1}\right)(x)<\infty .
$$

For $f \in \mathcal{L}_{1}(H, \mathcal{C}, \lambda)$, we define

$$
\int_{H} f \mathrm{~d} \mu \triangleq \int_{\mathbb{B}} x \mathrm{~d}\left(\lambda \circ f^{-1}\right)(x) .
$$

For actual calculations of probabilities of events in engineering application, one must restrict attention to a subclass of $\mathcal{L}(H, \mathcal{C}, \lambda)$. From now on $\lambda$ will denote a cylinder probability, unless mentioned otherwise. 
DEFINITION 2.2. Let $f: H \rightarrow H^{\prime}$ be Borel measurable and $\left\{P_{n}\right\}$ be a sequence of monotonically increasing finite-dimensional projections on $H$ converging strongly to the identity, which we denote by $P_{n} \uparrow I$. We say that $f \in \tilde{\mathcal{L}}\left(H, \mathcal{C}, \lambda ; H^{\prime}\right)$ if the sequence $\left\{f_{P_{n}}\right\}$ is Cauchy in $\lambda$-measure and

$$
C\left(h^{\prime}\right)=\lim _{n \rightarrow \infty} \int_{H} \exp \left\{\left[f_{P_{n}}(h), h^{\prime}\right]\right\} \mathrm{d} \lambda(h)
$$

is independent of the sequence $\left\{P_{n}\right\}$ chosen. It can be shown that $\widetilde{\mathcal{L}}\left(H, \mathcal{C}, \lambda ; H^{\prime}\right)$ is a subclass of $\mathcal{L}\left(H, \mathcal{C}, \lambda ; H^{\prime}\right)$. Elements of $\tilde{\mathcal{L}}\left(H, \mathcal{C}, \lambda ; H^{\prime}\right)$ will be called $H^{\prime}$-valued $\lambda$-physical random variables. When the cylinder probability is $\mu$, we refer to this class as $H^{\prime}$-valued physical random variables; or, p.r.v.'s for short. If $H^{\prime}=\mathbb{R}$, we denote the corresponding class of $\lambda$-p.r.v.'s by $\tilde{\mathcal{L}}(H, \mathcal{C}, \lambda)$.

For subsequent use we need the following:

DEFINITION 2.3. Suppose that, in Definition $2.2,\left\{f_{P_{n}}\right\}$ is actually $L_{2}$-Cauchy in $\lambda$-measure. We denote this subclass of $\widetilde{\mathcal{L}}\left(H, \mathcal{C}, \lambda ; H^{\prime}\right)$ by $\widetilde{\mathcal{L}}^{2}\left(H, \mathcal{C}, \lambda ; H^{\prime}\right)$. When $H^{\prime}=\mathbb{R}$, we denote this subclass for short by $\widetilde{\mathcal{L}}^{2}(\lambda)$. We now introduce a Gaussian white noise in our framework.

DEFINITION 2.4. The identity map $I:(H, \mathcal{C}, \mu) \rightarrow(H, \mathcal{C})$ is called a Gaussian white noise, or simply, a white noise.

One major difficulty of the 'finitely additive' white noise theory is that there is no complete characterization yet of a p.r.v. A useful sufficient condition for a mapping $f: H \rightarrow H^{\prime}$ to be a p.r.v. is given below. For this, we introduce some definitions.

DEFINITION 2.5. A function $f: H \rightarrow H^{\prime}$ is said to be continuous with respect to the $S$-topology at $x \in H$, or simply, $S$-continuous at $x$, if for any $\varepsilon>0$ there exists a Hilbert-Schmidt operator $L_{\varepsilon}(x): H \rightarrow H$ such that

$$
\left\|L_{\varepsilon}(x)(x-y)\right\|<1 \Longrightarrow\|f(x)-f(y)\|<\varepsilon .
$$

If the above result holds for $x, y \in U \subset H$ with $L_{\varepsilon}(x)$ independent of $x, f$ is then said to be uniformly $S$-continuous on $U$. If $U=H$, we simply say that $f$ is uniformly $S$-continuous.

A sufficient condition for functions to define p.r.v.'s is guaranteed by a weaker notion, called uniform $S$-continuity around the origin, which we introduce next.

DEFINITION 2.6. A function $f: H \rightarrow H^{\prime}$ is said to be uniformly $S$-continuous around the origin (u.s.c.a.o.) if $f$ is uniformly $S$-continuous on sets

$$
U_{n}=\left\{x \in H \mid\left\|L_{n} x\right\| \leqslant 1\right\},
$$

where $\left\{L_{n}\right\}_{1}^{\infty}$ is a sequence of Hilbert-Schmidt operators such that

$$
\left\|L_{n}\right\|_{\text {H.S. }} \longrightarrow 0 \text { as } n \rightarrow \infty \text { and } \bigcup_{n=1}^{\infty} U_{n}=H \text {. }
$$


A map uniformly $S$-continuous is obviously u.s.c.a.o. as may be seen by defining $L_{n}=\frac{1}{n} L$. A sufficient condition for a map to be a p.r.v. is given by (see [11]):

PROPOSITION 2.7. A sufficient condition for a function $f: H \rightarrow H^{\prime}$ to be a p.r.v. is that it be u.s.c.a.o.

Recently, an important characterization of maps which are u.s.c.a.o. has been given by de Santis et al. [22].

PROPOSITION 2.8. A function $f: H \rightarrow H^{\prime}$ is u.s.c.a.o. if and only if there exists a Hilbert-Schmidt operator $L: H \rightarrow H$ and a continuous function $g: H \rightarrow H^{\prime}$ such that

$$
f=g \circ L
$$

We conclude this discussion of p.r.v.'s by giving some examples [4].

\section{EXAMPLES.}

(1) Let $L: H \rightarrow H^{\prime}$ be linear and bounded. Then $L$ is a p.r.v. if and only if $L$ is Hilbert-Schmidt.

(2) $f: H \rightarrow \mathbb{R}$ is defined by $f(h)=[L h, h]$, where $L: H \rightarrow H$ is linear and bounded. Then $f$ is a p.r.v. if and only if $\left(L+L^{*}\right)$ is a trace-class operator.

In Section 4, we give generalization of Example 2 above yielding a much larger class of nonlinear p.r.v.'s. From Example 1 above and Definition 2.4, we conclude that a white noise is never a p.r.v. From Example 2, we also conclude that $f: H \rightarrow \mathbb{R}$ defined by $f(h)=\|h\|^{2}$ is also not a p.r.v. Although a white noise is never a p.r.v. it is a weak physical random variable, which we introduce next.

DEFINITION 2.9. A function $f: H \rightarrow H^{\prime}$ is called a $\lambda$-weak random variable if, for all $h^{\prime} \in H^{\prime}$, the function $f_{h^{\prime}}: H \rightarrow \mathbb{R}$ defined by $f_{h^{\prime}}(h)=\left[f(h), h^{\prime}\right]^{\prime}$, with $[\cdot, \cdot]^{\prime}$ the inner product in $H^{\prime}$, is a $\lambda$-random variable. The definition of $\lambda$-physical weak random variable is similar.

The next difficulty one encounters in this 'finitely additive' white noise theory is a suitable definition of conditional expectation which plays the central role in signal estimation. This is particularly so because of no natural role of $\sigma$-algebras in our setup. To define conditional expectation properly, we first introduce the useful notion of a representation of a cylinder probability and the corresponding lifting into the representation space. In fact, this is how Gross [12] defined a weak distribution and showed that it is equivalent to a cylinder probability (or, more generally, a cylinder measure). A representation ( $L, \Pi_{0}$ ) of the cylinder probability $\lambda$ is a mapping $L$ from $H$ into the class of all random variables on some countably additive probability space $\left(\Omega_{0}, \mathcal{A}_{0}, \Pi_{0}\right)$ such that

$$
L\left(\alpha_{1} h_{1}+\alpha_{2} h_{2}\right)=\alpha_{1} L\left(h_{1}\right)+\alpha_{2} L\left(h_{2}\right) \quad\left(\Pi_{0} \text {-a.s. }\right)
$$


and

$$
\lambda\left(h \mid\left[h, h_{1}\right] \leqslant x\right)=\Pi_{0}\left(L\left(h_{1}\right) \leqslant x\right)
$$

for all $x \in \mathbb{R}$.

DEFINITION 2.10. A function $f: H \rightarrow H^{\prime}$ is said to be a cylinder (tame) function if $f$ can be expressed as

$$
f(h)=f_{0}\left(\left[h, e_{1}\right], \ldots,\left[h, e_{n}\right]\right)
$$

for $e_{1}, \ldots, e_{n} \in H$ and $f_{0}: \mathbb{R}^{n} \rightarrow H^{\prime}$. If $\left(L, \Pi_{0}\right)$ is a representation of a cylinder probability $\lambda$, then for any such $f$ we define

$$
R_{\lambda}(f) \triangleq f_{0}\left(L\left(e_{1}\right), \ldots, L\left(e_{n}\right)\right) .
$$

Suppose now that $f \in \mathcal{L}\left(H, \mathcal{C}, \lambda ; H^{\prime}\right)$. Then the net $\left\{R_{\lambda}\left(f_{P}\right) \mid P \in \mathcal{P}\right\}, f_{P}$ a cylinder function as defined in (2), can be shown to converge in probability. The limit is called the $\lambda$-lifting of $f$ and is denoted by $R_{\lambda}(f)$. It can be proved that

$$
\lambda \circ f^{-1}=\Pi_{0} \circ\left[R_{\lambda}(f)\right]^{-1}
$$

and, as a consequence, for $f \in \mathcal{L}_{1}(H, \mathcal{C}, \lambda)$,

$$
\int_{H} f \mathrm{~d} \lambda=\int_{\Omega_{0}} R_{\lambda}(f) \mathrm{d} \Pi_{0} .
$$

We are now in a position to introduce the notion of conditional expectation. Let $\phi: H \rightarrow H^{\prime}$ be a $\lambda$-weak random variable and $\left(L, \Pi_{0}\right)$ be a representation of the cylinder probability $\lambda$. Define $L^{\prime}$ on $H^{\prime}$ by

$$
L^{\prime}\left(h^{\prime}\right) \triangleq R_{\lambda}\left(\left[\phi, h^{\prime}\right]^{\prime}\right)
$$

Then for any cylinder set $C^{\prime} \in \mathcal{C}^{\prime}$ of the form

$$
C^{\prime}=\left\{k^{\prime} \in H^{\prime} \mid\left(\left[k^{\prime}, h_{1}\right]^{\prime}, \ldots,\left[k^{\prime}, h_{r}\right]^{\prime}\right) \in B\right\},
$$

$h_{1}, \ldots, h_{r} \in H^{\prime}$ and $B$ a Borel set in $\mathbb{R}^{r}$, define

$$
\lambda^{\prime}\left(C^{\prime}\right)=\Pi_{0}\left(\left(L^{\prime}\left(h_{1}\right), \cdots, L^{\prime}\left(h_{r}\right)\right) \in B\right) .
$$

$\lambda^{\prime}$ thus defined is a cylinder probability on $\left(H^{\prime}, \mathcal{C}^{\prime}\right)$ and is denoted by $\lambda \circ \phi^{-1}$. Furthermore, $\left(L^{\prime}, \Pi_{0}\right)$ is a representation of $\lambda \circ \phi^{-1}$ and is called the representation induced by $\phi$. Let $R_{\lambda^{\prime}}$ be the corresponding lifting of $\lambda^{\prime}$ and define

$$
\mathcal{U}(\phi) \equiv \mathcal{U}(H, \mathcal{C}, \lambda ; \phi) \triangleq\left\{g \in \mathcal{L}\left(H^{\prime}, \mathcal{C}^{\prime}, \lambda^{\prime}\right) \mid R_{\lambda^{\prime}}(g)=R_{\lambda}(g \circ \phi)\right\} .
$$


If $g \in \mathcal{U}(\phi)$, it can be shown that

$$
\lambda^{\prime} \circ g^{-1}=\lambda \circ(g \circ \phi)^{-1} \text {. }
$$

We need to introduce the class $\mathcal{U}(\phi)$ because it is not yet clear as to whether (8) holds for all $g \in \mathcal{L}\left(H^{\prime}, \mathcal{C}^{\prime}, \lambda^{\prime}\right)$.

DEFINITION 2.11. Let $\phi: H \rightarrow H^{\prime}$ be a weak random variable. For $f \in \mathcal{L}(H, \mathcal{C}, \lambda)$, if there exists $g \in \mathcal{U}(\phi)$ such that

$$
\int_{H} f 1_{C^{\prime}}(\phi) \mathrm{d} \lambda=\int_{H} g(\phi) \mathbf{1}_{C^{\prime}}(\phi) \mathrm{d} \lambda
$$

for all $C^{\prime} \in \mathcal{C}^{\prime}$, then $g$ is defined to be the conditional expectation of $f$ given $\phi$, denoted by $E_{\lambda}[f \mid \phi]$. Details may be found in [15].

\section{Stochastic Differential Equations with White Noise Inputs}

Let $f: \mathbb{R}^{n} \rightarrow \mathbb{R}^{n}$ be a Lipschitz function. For $x_{0} \in \mathbb{R}^{n}$ and $h \in H$, consider the following differential equation

$$
\frac{\mathrm{d} x_{t}(h)}{\mathrm{d} t}=f\left(x_{t}(h)\right)+\eta_{t}(h)
$$

where $\eta$ defined by $\eta_{t}(h)=h(t)$ is a white noise on $H$. Note that (9) is equivalent to

$$
x_{t}(h)=x_{0}+\int_{0}^{t} f\left(x_{\tau}(h)\right) \mathrm{d} \tau+\int_{0}^{t} \eta_{\tau}(h) \mathrm{d} \tau
$$

Let $B=C\left([0, t] ; \mathbb{R}^{n}\right)$. Then $B$ is a separable Banach space. It can be shown [2] that (9) or (10) has a unique solution and, furthermore,

$$
x_{t} \in \mathcal{L}(H, \mathcal{C}, \mu) \quad \text { and } \quad x \in \mathcal{L}(H, \mathcal{C}, \mu ; B) .
$$

This direct modeling of the state process, without taking recourse to the integrated form, is appealing. The question is whether the solution process of (9) is, indeed, Markov. Markov process has to be defined properly because of the inability to work with $\sigma$-algebras in our set-up. We may resolve this difficulty by introducing 'past' and 'future' of the process in an appropriate manner. For this we introduce two Hilbert spaces

$$
H_{1}=L_{2}\left([0, s] ; \mathbb{R}^{n}\right) \quad \text { and } \quad H_{2}=L_{2}\left([s, T] ; \mathbb{R}^{n}\right) \text {. }
$$

Let us define $Q_{1}: H \rightarrow H_{1}$ and $Q_{2}: H \rightarrow H_{2}$ by

$$
\begin{array}{ll}
Q_{1}(h)(t)=h(t), & 0 \leqslant t \leqslant s ; \\
Q_{2}(h)(t)=h(t), & s \leqslant t \leqslant T .
\end{array}
$$


Intuitively, the mapping $Q_{1}$ captures the 'past' and $Q_{2}$ captures the 'future' of the process. Both $Q_{1}$ and $Q_{2}$ are weak random variables. In analogy with the usual theory, we may define a process $\left\{x_{t}, 0 \leqslant t \leqslant T\right\}$ to be Markov if, for $t \geqslant s$, $E_{\mu}\left[x_{t} \mid Q_{1}\right]$ is a function of $x_{s}$ only. To prove this kind of results, we need a form of Fubini theorem whose proof is by no means obvious.

Let us define $Q: H_{1} \times H_{2} \rightarrow H$ by

$$
Q\left(h_{1}, h_{2}\right)(t)= \begin{cases}h_{1}(t), & t \leqslant s \\ h_{2}(t), & t>s .\end{cases}
$$

We have already mentioned that $Q_{1}$ and $Q_{2}$, defined above, are weak random variables. Then $\mu_{1}=\mu \circ Q_{1}^{-1}$ and $\mu_{2}=\mu \circ Q_{2}^{-1}$ are Gauss cylinder probabilities on $H_{1}$ and $\mathrm{H}_{2}$, respectively. We now have the following form of Fubini theorem:

THEOREM 3.1. Let $f: H \rightarrow \mathbb{R}$ belong to $\mathcal{L}_{1}(H, \mathcal{C}, \mu)$ and satisfy $f=f^{\sim} \circ \gamma$ for some real-valued bounded continuous function $f^{\sim}$ on $B$ and $\gamma: H \rightarrow B$ be defined by

$$
\gamma(h)(t)=\int_{0}^{t} h(s) \mathrm{d} s, \quad h \in H .
$$

Then

$$
\int f(h) \mathrm{d} \mu(h)=\iint f\left(Q\left(h_{1}, h_{2}\right)\right) \mathrm{d} \mu_{2}\left(h_{2}\right) \mathrm{d} \mu_{1}\left(h_{1}\right) .
$$

Furthermore, if

$$
f_{1}\left(h_{1}\right) \triangleq \int f\left(Q\left(h_{1}, h_{2}\right)\right) \mathrm{d} \mu_{2}\left(h_{2}\right)
$$

then

$$
E_{\mu}\left[f \mid Q_{1}\right]=f_{1} \circ Q_{1}
$$

Proof. See [15, Theorem 3].

Using Theorem 3.1, we may establish the Markov property as follows:

THEOREM 3.2. Let $g$ be a bounded continuous function on $\mathbb{R}^{n}$ and $\left\{x_{t}, 0 \leqslant t \leqslant T\right\}$ be the solution of (9). Then, for $t \geqslant s$,

$$
E_{\mu}\left[g\left(x_{t}\right) \mid Q_{1}\right]=g_{1}\left(x_{s}\right),
$$

where

$$
g_{1}(x)=\int g\left(\Gamma_{s t}\left(x, h_{2}\right)\right) \mathrm{d} \mu_{2}\left(h_{2}\right)
$$


with $x_{t}^{\prime} \triangleq \Gamma_{s t}\left(x, h_{2}\right)$ being the unique solution of

$$
x_{t}^{\prime}=x+\int_{s}^{t} f\left(x_{\tau}^{\prime}\right) \mathrm{d} \tau+\int_{s}^{t} \eta_{\tau}(h) \mathrm{d} \tau, \quad t \geqslant s .
$$

Proof. We repeat the proof given in [2]. Let $f(h) \triangleq g\left(x_{t}(h)\right)$ and $f^{\sim}(Z) \triangleq$ $g\left(X_{t}(Z)\right)$, where $\left\{X_{t}(Z)\right\}$ is the unique solution of

$$
X_{t}(Z)=x_{0}+\int_{0}^{t} f\left(X_{\tau}(Z)\right) \mathrm{d} \tau+Z(t)
$$

with $Z \in B=C\left([0, T] ; \mathbb{R}^{n}\right)$. Then $f^{\sim}$ is a continuous function and $f=f^{\sim} \circ \gamma$, with $\gamma$ defined by (11). Therefore, by Theorem 3.1,

$$
E_{\mu}\left[f \mid Q_{1}\right]=f_{1} \circ Q_{1}
$$

exists. We now show that

$$
f_{1} \circ Q_{1}=g_{1}\left(x_{1}\right)
$$

For $h_{1} \in H_{1}$, let $\xi_{\tau}\left(h_{1}\right), \tau \leqslant s$, be the solution of

$$
\xi_{\tau}=x_{0}+\int_{0}^{\tau} f\left(\xi_{\sigma}\right) \mathrm{d} \sigma+\int_{0}^{\tau} h_{1}(\sigma) \mathrm{d} \sigma .
$$

Then

$$
x_{s}(h)=\xi_{s}\left(Q_{1} h\right) .
$$

Also

$$
x_{t}\left(Q\left(h_{1}, h_{2}\right)\right)=\Gamma_{s t}\left(\xi_{s}\left(h_{1}\right), h_{2}\right) .
$$

Therefore,

$$
\begin{aligned}
f_{1}\left(h_{1}\right) & =\int g\left(\Gamma_{s t}\left(\xi_{s}\left(h_{1}\right), h_{2}\right)\right) \mathrm{d} \mu_{2}\left(h_{2}\right) \\
& =g_{1}\left(\xi_{s}\left(h_{1}\right)\right) .
\end{aligned}
$$

Then

$$
f_{1}\left(Q_{1} h\right)=g_{1}\left(\xi_{s}\left(Q_{1} h\right)\right)=g_{1}\left(x_{s}(h)\right)
$$

establishing the Markov property (13). 
Once we are able to model the state process directly as the solution of a differential equation with a white noise input in a physically meaningful manner, it becomes possible to study the nonlinear filtering problem with correlated state and observation noises, using the 'finitely additive' white noise approach. We illustrate the main ideas by considering only the scalar case of $n=1$. Details may be found in [2].

We take $H=L_{2}\left([0, T] ; \mathbb{R}^{2}\right)$ and for $h \in H$ expressed as $h(t)=\left(h_{1}(t) h_{2}(t)\right)^{\prime}$, define

$$
\eta_{1 t}(h) \triangleq h_{1}(t) \quad \text { and } \quad \eta_{2 t}(h) \triangleq h_{2}(t) .
$$

Suppose that the signal process $\left\{x_{t}(h)\right\}$ is given by

$$
\begin{aligned}
& \dot{x}_{t}(h)=f\left(x_{t}(h)\right)+\alpha \eta_{1 t}(h)+\delta \eta_{2 t}(h), \\
& x_{0}(h)=x_{0}
\end{aligned}
$$

where $f: \mathbb{R} \rightarrow \mathbb{R}$ is a bounded Lipschitz function and $\alpha>0, \delta>0$ are real numbers, while the observation process $\left\{y_{t}(h)\right\}$ is modelled as

$$
y_{t}(h)=g\left(x_{t}(h)\right)+\eta_{2 t}(h)
$$

where $g$ is assumed to be a twice continuously differentiable function on $\mathbb{R}$ such that $g$ and $g^{\prime}$ are bounded. Nonlinear filtering problem consists in obtaining a formula for

$$
\pi_{t}(\phi, y) \triangleq E_{\mu}\left[\phi\left(x_{t}\right) \mid Q_{t} y\right]
$$

where $Q_{t}: H \rightarrow H$ is defined by $\left(Q_{t} h\right)(s)=h(s) \mathbf{1}_{[0, t]}(s)$. It is intuitively clear that the right-hand side of (16) is the 'white noise' analog of the expression $E\left[\phi\left(x_{t}\right) \mid\right.$ $\left.y_{s} ; 0 \leqslant s \leqslant t\right]$ calculated in the usual set-up. To calculate $\pi_{t}(\phi, y)$ as defined by (16) we introduce a new process $\left\{z_{t}(h)\right\}$ by

$$
\begin{aligned}
& \dot{z}_{t}(h)=\dot{x}_{t}(h)-\delta y_{t}(h), \\
& z_{0}(h)=x_{0} .
\end{aligned}
$$

Then

$$
z_{t}(h)=v_{t}\left(\eta_{1}(h), y(h)\right)
$$

where, for $\bar{h}_{1}, \bar{h}_{2} \in L_{2}([0, T] ; \mathbb{R}), v_{t} \equiv v_{t}\left(\bar{h}_{1}, \bar{h}_{2}\right)$ is the unique solution of

$$
\begin{aligned}
v_{t}= & x_{0}+\int_{0}^{t}\left[f\left(v_{s}+\delta \hat{\vec{h}}_{2}(s)\right)-\delta g\left(v_{s}+\delta \hat{\bar{h}}_{2}(s)\right)\right] \mathrm{d} s+ \\
& +\alpha \int_{0}^{t} \bar{h}_{1}(s) \mathrm{d} s
\end{aligned}
$$


with

$$
\hat{\bar{h}}_{2}(s) \triangleq \int_{0}^{s} \bar{h}_{2}(\tau) \mathrm{d} \tau .
$$

Let $\bar{\mu}$ be the canonical Gauss measure on $L_{2}([0, T] ; \mathbb{R})$. For $\bar{h}_{2} \in L_{2}([0, T] ; \mathbb{R})$, define

$$
\begin{aligned}
\sigma_{t}\left(\phi, \bar{h}_{2}\right)= & \int_{L_{2}([0, T\} ; \mathbb{I})} \phi\left(v_{t}\left(\bar{h}_{1}, \bar{h}_{2}\right)+\delta \hat{\bar{h}}_{2}(t)\right) \\
& \times \exp \left\{\int_{0}^{t} g\left(v_{s}\left(\bar{h}_{1}, \bar{h}_{2}\right)+\delta \hat{\bar{h}}_{2}(s)\right) \bar{h}_{2}(s) \mathrm{d} s-\right. \\
& \left.-\frac{1}{2} \int_{0}^{t}\left(\alpha g^{\prime}+g^{2}\right)\left(v_{s}\left(\bar{h}_{1}, \bar{h}_{2}\right)+\delta \hat{\bar{h}}_{2}(s)\right) \mathrm{d} s\right\} \mathrm{d} \bar{\mu}\left(\bar{h}_{1}\right) .
\end{aligned}
$$

Then the robust nonlinear filtering formula for correlated signal and noise processes is given by

$$
E_{\mu}\left[\phi\left(x_{t}\right) \mid Q_{t} y\right]=\frac{\sigma_{t}(\phi, y)}{\sigma_{t}(1, y)}
$$

For a proof of this result see Theorem 5 of [2].

\section{Radon-Nikodym Theorem of Induced Finitely Additive Measures}

We begin with some definitions.

DEFINITION 4.1. A cylinder probability $\nu$ is said to be absolutely continuous with respect to another cylinder probability $\lambda$ if, for any $\varepsilon>0$, there exists a $\delta>0$ such that for any cylinder set $C \in \mathcal{C}, \lambda(C)<\delta \Rightarrow \nu(C)<\varepsilon$.

Note that this does not imply the existence of the Radon-Nikodym derivative as in the countably additive framework. The following definition gives a natural way of introducing Radon-Nikodym derivatives for finitely additive measures.

DEFINITION 4.2. Suppose that $\nu$ and $\lambda$ are cylinder probabilities on $(H, \mathcal{C})$ and $\nu$ is absolutely continuous with respect to $\lambda$. We say that $f$ is the Radon-Nikodym derivative of $\nu$ with respect to $\lambda$ if $f$ is a $\lambda$-p.r.v. and, for any sequence of projections $P_{n} \in \mathcal{P}$ increasing strongly to the identity,

$$
\nu(C)=\lim _{n \rightarrow \infty} \int_{C} f\left(P_{n} h\right) \mathrm{d} \lambda(h)
$$

uniformly in $C \in \mathcal{C}$ and is written as

$$
f(h)=\frac{\mathrm{d} \nu}{\mathrm{d} \lambda}(h)
$$

for $h \in H$. 
The next two theorems deal with the existence of Radon-Nikodym derivatives in the context of cylinder probabilities.

THEOREM 4.3. Let $\nu$ and $\lambda$ be two cylinder probabilities on $H$ and let $\nu$ be absolutely continuous with respect to $\lambda$. Then there exists a sequence $\left\{\phi_{n}\right\}$ of cylinder functions with

$$
\int_{H}\left|\phi_{n}(h)\right| \mathrm{d} \lambda(h)<\infty
$$

such that

$$
\nu(C)=\lim _{n \rightarrow \infty} \int_{C} \phi_{n}(h) \mathrm{d} \lambda(h)
$$

uniformly in $C \in \mathcal{C}$.

Proof. The proofs of this and the following theorem are based on a method used by Fefferman [10]. See [1] for details.

THEOREM 4.4. Let $\left\{P_{n}\right\}$ be an increasing sequence of projections in $\mathcal{P}$ converging strongly to the identity. Let $\nu$ and $\lambda$ be as in Theorem 4.3 and define $\nu_{n}=\left.\nu\right|_{\mathcal{C}_{n}}$ and $\lambda_{n}=\left.\lambda\right|_{\mathcal{C}_{P_{n}}}$. Suppose there exists a function $f(\cdot)$ such that

$$
f\left(P_{n} h\right)=\frac{\mathrm{d} \nu_{n}}{\mathrm{~d} \lambda_{n}}\left(P_{n} h\right)
$$

Then

$$
\nu(C)=\lim _{n \rightarrow \infty} \int_{C} f\left(P_{n} h\right) \mathrm{d} \lambda(h)
$$

uniformly in $C \in \mathcal{C}$.

Proof. See [1].

We want to determine in this section Radon-Nikodym derivatives of finitely additive measures induced by nonlinear transformations on $H$ with respect to the canonical Gauss measure $\mu$. We want to use Theorem 4.4 for this purpose. From Definition 4.2 we see that it is then necessary to have a characterization for $f$ to be a p.r.v. As mentioned earlier, no characterization for an arbitrary $f$ to be a p.r.v. is yet available. We first give a sufficient condition for a nonlinear mapping $f: H \rightarrow \mathbb{R}$ defined by $f(h)=[M(h), h], h \in H$, to be a p.r.v., where $M: H \rightarrow H$ is a nonlinear map. Fortunately, for studying Radon-Nikodym derivatives of finitely additive measures induced by nonlinear transformations, only functions $f$ of the form mentioned above are encountered.

THEOREM 4.5. Let $M: H \rightarrow H$ be a nonlinear mapping which satisfies the following hypotheses:

(1) $\left\|M_{x} y\right\| \leqslant\|A y\|, x, y \in H$, where $A$ is a Hilbert-Schmidt operator from $H$ into $H$, independent of $x$,

(2) Let $\left(M_{x}+M_{x}^{*}\right)$ be trace class and $\sup _{x}\left\|M_{x}+M_{x}^{*}\right\|_{1}<\infty$.

Then $[M(h), h], h \in H$, is a $\tilde{\mathcal{L}}^{2}(\mu)$ p.r.v. 
Outline of proof. We first consider the function $f: H \rightarrow \mathbb{R}$ defined by

$$
f(h)=[M(h), h]-\frac{1}{2} \operatorname{Tr}\left(M_{h}+M_{h}^{*}\right)
$$

and show that $f$ is a $\widetilde{\mathcal{L}}^{2}(\mu)$ p.r.v. The proof is based upon the usual procedure of considering finite dimensional approximations and taking limits. At this step we make use of a result shown for standard Gauss measures on finite dimensional spaces by Ramer [21]. It states that, if $f: \mathbb{R}^{n} \rightarrow \mathbb{R}^{n}$ is a $C^{1}$-map such that $|f(x)|$ and $\left\|f_{x}\right\|_{2} \in L^{2}\left(\mathbb{R}^{n}, \mu^{n}\right)$ with $\mu^{n}$ the standard Gauss measure on $\mathbb{R}^{n}$ with variance parameter 1 , then

$$
\int_{\mathbb{R}^{n}}\left([f(x), x]-\operatorname{Tr} f_{x}\right)^{2} \mathrm{~d} \mu^{n}(x) \leqslant \int_{\mathbb{\mathbb { R }}^{n}}\left(|f(x)|^{2}+\left\|f_{x}\right\|_{2}^{2}\right) \mathrm{d} \mu^{n}(x) .
$$

From our assumptions, we may also prove (see Lemma 3.4 in [3]) that $\operatorname{Tr}\left(M_{h}+M_{h}^{*}\right)$ is a $\widetilde{\mathcal{L}}^{2}(\mu)$ p.r.v. Combining these two, we get the desired result. Details may be found in [3].

We are now in a position to study Radon-Nikodym derivatives as mentioned above. Let $M: H \rightarrow H$ be a nonlinear map satisfying the hypotheses (1) and (2) of Theorem 4.5. Let $M_{h}$ denote the Fréchet derivative of $M$ at $h \in H$ and suppose that it is defined for all $h \in H$ and is Hilbert-Schmidt, Volterra (quasi-nilpotent). Then $\left(I-M_{h}\right)^{-1}$ exists and by the result in Dunford and Schwartz ([8, p. 1039]), we have

$$
\left\|\left(I-M_{h}\right)^{-1}\right\| \leqslant \exp \left\{\frac{1+\left\|M_{h}\right\|_{2}^{2}}{2}\right\} \leqslant \exp \left\{\frac{1+\|A\|_{2}^{2}}{2}\right\} .
$$

Hence, by using Hadamard's theorem (see Berger [6, p. 222]), it follows that ( $I-$ $M)^{-1}=I+K$ exists. Note also that hypothesis (2) of Theorem 4.5 implies that the Fréchet derivative of $K$, denoted $K_{h}$, also satisfies condition (2) of Theorem 4.5 for another Hilbert-Schmidt operator $B$ defined by

$$
B=\exp \left(\frac{1+\|A\|_{2}^{2}}{2}\right) A
$$

This follows from the result (see Balakrishnan [4]):

$$
K_{h}=\left(I-M_{z(h)}\right)^{-1} M_{z(h)},
$$

where $z(h)=(I-M)^{-1}(h)$ for all $h \in H$. Furthermore, $M_{h}$ being Hilbert-Schmidt and Volterra, by the mean value theorem, $K(h)$ is uniformly $S$-continuous with

$$
\int_{H}\|K(h)\|^{2} \mathrm{~d} \mu(h)<\infty .
$$

We can now state the following theorem: 
THEOREM 4.6. Let $M: H \rightarrow H$ be a nonlinear map satisfying the hypotheses (1) and (2) of Theorem 4.5. Then $T \triangleq(I-M)^{-1}: H \rightarrow H$ induces a cylinder probability $\mu_{T}$ on $H$ which is absolutely continuous with respect to $\mu$ on $H$ and

$$
\frac{\mathrm{d} \mu_{T}}{\mathrm{~d} \mu}(h)=g(h)
$$

where

$$
g(h)=\exp \left\{[M(h), h]-\frac{1}{2}\|M(h)\|^{2}-\frac{1}{2} \operatorname{Tr}\left(M_{h}+M_{h}^{*}\right)\right\}
$$

for $h \in H$. Equivalently, for any cylinder set $C \in \mathcal{C}$,

$$
\mu_{T}(C)=\lim _{n \rightarrow \infty} \int_{C} g\left(P_{n} h\right) \mathrm{d} \mu(h)
$$

uniformly in $C \in \mathcal{C}$, as $P_{n}$ converges strongly to $I$.

Outline of proof. Consider the sequence of finite dimensional maps:

$$
S_{n}=\left(I-P_{n} M P_{n}\right): P_{n} H \longrightarrow P_{n} H .
$$

The theorem is proved via the following steps:

(1) We show that $S_{n}^{-1}=T_{n}$ exists and $T_{n}=I+P_{n} N P_{n}$ with $\left\|P_{n} N_{P_{n} x} P_{n} y\right\| \leqslant$ $k\left\|A P_{n} y\right\|$ and, denoting the induced cylinder measure of $T_{n}$ by $\mu_{T_{n}}$, we show that the corresponding characteristic function converges to the characteristic function of $\mu_{T}$ as $P_{n}$ converges strongly to $I$.

(2) Then using the Jacobian formula for finite dimensions we show that

$$
\frac{\mathrm{d} \mu_{T_{n}}}{\mathrm{~d} \mu}\left(P_{n} h\right)=f_{n}\left(P_{n} h\right) .
$$

(3) We then show that $f_{n}\left(P_{n} h\right)$ converges to $g(h)$ in $L_{1}(\mu)$ and noting that $g(h)$ is a p.r.v. (by Theorem 4.5 and part of Lemma 3.4 in [3]), we complete the proof via Theorem 4.4 .

Details may be found in [3].

We now use this result to obtain directly the Wong-Zakai correction term. Let us go back to equation (9) in scalar form:

$$
\frac{\mathrm{d} x_{t}(h)}{\mathrm{d} t}=f\left(x_{t}(h)\right)+\eta_{t}(h), \quad h \in H \equiv L_{2}[0, T],
$$

with $f: \mathbb{R} \rightarrow \mathbb{R}, x_{0} \in \mathbb{R}$ given and $\eta$ is a white noise on $H$. We make the following assumption:

(A1) $f \in C^{1}$ and $f^{\prime}$ is bounded.

Then we can state the following theorem: 
THEOREM 4.7. Under assumption (A1), the process $\dot{x}$ induces a cylinder probability, denoted by $\mu_{\dot{x}}$ on $H$, which is absolutely continuous with respect to the canonical Gauss measure $\mu$ on $H$ and the Radon-Nikodym derivative is given by:

$$
\frac{\mathrm{d} \mu_{\dot{x}}}{\mathrm{~d} \mu}(h)=\exp \left\{[f(L h), h]-\frac{1}{2}\|f(L h)\|^{2}-\frac{1}{2} \int_{0}^{T} f^{\prime}\left(\int_{0}^{t} h(s) \mathrm{d} s\right) \mathrm{d} t\right\},
$$

where $L$ is a linear Volterra operator from $H$ into $H$ given by

$$
(L h)(t)=g(t) ; \quad g(t)=\int_{0}^{t} h(s) \mathrm{d} s .
$$

Proof. See [3].

We now study one important consequence of the preceding theorem. From Theorem 4.7 the likelihood functional of the process $\left\{\dot{x}_{t}, 0 \leqslant t \leqslant T\right\}$ is given by

$$
\frac{\mathrm{d} \mu_{\dot{x}}}{\mathrm{~d} \mu}(\dot{x})=\exp \left\{-\frac{1}{2}\left\{\int_{0}^{T} f\left(x_{t}\right)^{2} \mathrm{~d} t-2 \int_{0}^{T} f\left(x_{t}\right) \dot{x}_{t} \mathrm{~d} t+\int_{0}^{T} f^{\prime}\left(x_{t}\right) \mathrm{d} t\right\}\right\} .
$$

We compare this expression for the likelihood functional with the corresponding result for the usual Wiener process model. The latter formula, obtained by Prohorov [20], is given by

$$
\frac{\mathrm{d} \mu_{x}}{\mathrm{~d} \mu_{w}}(x)=\exp \left\{-\frac{1}{2}\left\{\int_{0}^{T} f\left(x_{t}\right)^{2} \mathrm{~d} t-2 \int_{0}^{T} f\left(x_{t}\right) \mathrm{d} x_{t}\right\}\right\}
$$

where $\mu_{x}$ is the measure induced on $C[0, T]$ by the process $\left\{x_{t}, 0 \leqslant t \leqslant T\right\}$ satisfying the Itô stochastic differential equation:

$$
\mathrm{d} x_{t}=f\left(x_{t}\right) \mathrm{d} t+\mathrm{d} w_{t}
$$

and $\mu_{w}$ denotes the Wiener measure on $C[0, T]$. The second integral in the exponential expression on the right hand side of (30) is an Itô stochastic integral.

It is interesting to compare the two expressions (29) and (30). In the white noise formulation, the likelihood functional is the exact limit of the corresponding result for band-limited processes, as the band-width increases without bound. On the other hand, as Wong and Zakai [24] showed in the late sixties, the limit of integrals for band-limited processes converge to the corresponding stochastic integral only when corrected by a suitable term. In fact, from the result of Wong and Zakai, one obtains:

$$
\int_{0}^{T} f\left(x_{t}\right) \mathrm{d} x_{t}=\lim _{k \rightarrow \infty}\left[\int_{0}^{T} f\left(x_{t}^{k}\right) \dot{x}_{t}^{k} \mathrm{~d} t-\frac{1}{2} \int_{0}^{T} f^{\prime}\left(x_{t}^{k}\right) \mathrm{d} t\right],
$$


where $\left\{x_{t}^{k}, 0 \leqslant t \leqslant T\right\}$ satisfies

$$
\frac{\mathrm{d} x_{t}^{k}}{\mathrm{~d} t}=f\left(x_{t}^{k}\right)+n_{t}^{k}
$$

where $\left\{n_{t}^{k}, 0 \leqslant t \leqslant T\right\}$ approaches a Gaussian white noise in the limit (see Wong and Hajek [23] for details). This explains the difference between the expressions (29) and (30). It also highlights the advantage of working with the direct model of white noise when one has to handle real data. The process $\left\{x_{t}, 0 \leqslant t \leqslant T\right\}$, which is the solution of (31), is only an idealization. The real data that one observes in practice cannot be nondifferentiable everywhere, as the model (31) implies. Every measurement apparatus acts as a filter on the idealized data $\left\{\dot{x}_{t}, 0 \leqslant t \leqslant T\right\}$. When one has to evaluate the stochastic integral

$$
\int_{0}^{T} f\left(x_{t}\right) \mathrm{d} x_{t}
$$

$\mathrm{d} x_{t}$ is replaced by $\dot{x}_{t} \mathrm{~d} t$, where $\dot{x}_{t}$ is calculated from real data. In fact, for real data, $\dot{x}_{t}$ is band-limited, albeit with large band-width. By replacing $\mathrm{d} x_{t}$ by $\dot{x}_{t} \mathrm{~d} t$ will give us the wrong result and will have to be corrected by the Wong-Zakai correction term

$$
\left(-\frac{1}{2} \int_{0}^{T} f^{\prime}\left(x_{t}\right) \mathrm{d} t\right)
$$

in our case. In the white noise model this term already appears in the expression for the likelihood ratio and no such correction is necessary in this approach when handling real data. In technical terms we say that the finitely additive white noise theory always gives results in the robust form.

\section{A Representation Result for Nonlinear Filter Maps}

We again consider the nonlinear filtering problem studied in Section 3, but now in the more conventional situation when the state and observation noises are uncorrelated. Thus, in the scalar case, the signal process $\left\{x_{t}(h)\right\}$ evolves according as

$$
\begin{aligned}
& \dot{x}_{t}(h)=f\left(x_{t}(h)\right)+\eta_{1 t}(h), \\
& x_{0}(h)=x_{0},
\end{aligned}
$$

while the observation process $\left\{y_{t}(h)\right\}$ is given by

$$
y_{t}(h)=x_{t}(h)+\eta_{2 t}(h) \text {. }
$$

Here $h \in H=L_{2}\left([0, T] ; \mathbb{R}^{2}\right), h(t)=\left(h_{1}(t) h_{2}(t)\right)^{\prime}$ and $f: \mathbb{R} \rightarrow \mathbb{R}$ is a bounded Lipschitz function. Under these conditions, it can be shown (see [4]) that

$$
E_{\mu} \int_{0}^{T} x_{t}^{2} \mathrm{~d} t<\infty
$$


Following Kallianpur and Karandikar [15], the conditional expectation $E_{\mu}\left[x_{t} \mid Q_{t} y\right]$ exists and satisfies the Kallianpur-Striebel formula:

$$
\psi_{t}(y) \triangleq E_{\mu}\left[x_{t} \mid Q_{t} y\right]=\frac{\int_{\bar{H}} x_{t}\left(\bar{h}_{1}\right) B(t, x, y) \mathrm{d} \bar{\mu}\left(\bar{h}_{1}\right)}{\int_{\bar{H}} B(t, x, y) \mathrm{d} \bar{\mu}\left(\bar{h}_{1}\right)} .
$$

Here $Q_{t}: \bar{H} \triangleq L_{2}([0, T] ; \mathbb{R}) \rightarrow \bar{H}$ is as defined in Section $3, \bar{\mu}$ is the canonical Gauss measure on $\bar{H}$ and

$$
B(t, x, y) \triangleq \exp \left\{\int_{0}^{t} x_{s}\left(\bar{h}_{1}\right) y_{s} \mathrm{~d} s-\frac{1}{2} \int_{0}^{t}\left|x_{s}\left(\bar{h}_{1}\right)\right|^{2} \mathrm{~d} s\right\},
$$

where the integration is performed with $y$ treated as a parameter. The function $\psi_{t}(\cdot)$ is referred to as the nonlinear filter map. We can now prove the following result:

THEOREM 5.1. The conditional expectation $\psi_{t}(y)$ may be written as

$$
\psi_{t}(y)=\bar{\psi}_{t}(K y)
$$

where $\bar{\psi}_{t}$ maps $\bar{H}^{t} \triangleq L_{2}([0, t] ; \mathbb{R})$ into itself and $K: \bar{H}^{t} \rightarrow \bar{H}^{t}$ is a (linear) HilbertSchmidt operator.

Proof. We first prove that $\psi_{t}(\cdot)$ is uniformly $S$-continuous around the origin in $\bar{H}^{t}$ (see [17] for details). Then invoking Proposition 2.8 the result follows.

The property of uniform $S$-continuity around the origin of the nonlinear filter map leads to a simple proof of the approximation of nonlinear filter maps by finite 'Volterra' polynomials. For the definition of a Volterra polynomial, see [4]. It basically follows from a generalization of the classical Weierstrass theorem to Hilbert spaces [19]. In fact, we have

THEOREM 5.2. The function $\psi_{t}(\cdot)$ may be approximated by a finite Volterra polynomial on every bounded sphere in $\bar{H}^{t}$; that is, on the set $S_{n}=\left\{h \in \bar{H}^{t} \mid\|h\| \leqslant n\right\}$, there exists a Volterra polynomial $p_{t}^{N_{\epsilon}}(K h)$ of order $N_{\varepsilon}$ such that

$$
\sup _{h \in S_{n}}\left\|p_{t}^{N_{*}}(K h)-\psi_{t}(h)\right\|<\varepsilon
$$

with $K$ a Hilbert-Schmidt operator on $\bar{H}^{t}$.

Proof. See [17].

We now discuss a far-reaching consequence of Theorem 5.1. In a seminal paper, Benès [5] in 1981 showed the existence of finite dimensional filters for nonlinear filtering problems with the drift term satisfying a certain condition, usually referred to as the Beněs condition. It assumes that the nonlinear drift term $f(\cdot)$ satisfies

$$
f^{2}(x)+f^{\prime}(x)=v(x)
$$


where

$$
v(x)=a x^{2}+b x+c \quad \text { with } a>-1 .
$$

THEOREM 5.3. Under Beněs condition as stated above, the nonlinear filter is given by

$$
\psi_{t}(y)=\bar{\psi}_{t}(K y)
$$

where $\bar{\psi}_{t}: \bar{H}^{t} \rightarrow \bar{H}^{t}$ is strongly continuous and $K$ is a Hilbert-Schmidt operator related to a linear-Gaussian filtering problem.

Proof. Let $\bar{\mu}_{\dot{x}}^{t}$ be the cylinder measure induced by the process $\left\{\dot{x}_{s}, 0 \leqslant s \leqslant t\right\}$, satisfying (33), on $\bar{H}^{t}$, with $\bar{\mu}^{t}$ the canonical Gauss measure thereon. Since

$$
x_{s}=\int_{0}^{s} \dot{x}_{\sigma} \mathrm{d} \sigma=(L \dot{x})_{s}
$$

with the operator $L$ being Hilbert-Schmidt, Volterra, we may rewrite the numerator of (35) as

$$
\int_{\bar{H}} x_{t}\left(\bar{h}_{1}\right) B(t, x, y) \mathrm{d} \bar{\mu}\left(\bar{h}_{1}\right)=\int_{\bar{A}^{t}}(L \bar{h})_{t} B(t, L \bar{h}, y) \mathrm{d} \bar{\mu}_{\dot{x}}^{t}(\bar{h}) .
$$

We know from Theorem 4.7 that

$$
\frac{\mathrm{d} \bar{\mu}_{\grave{x}}^{t}}{\mathrm{~d} \bar{\mu}^{t}}(\bar{h})=\exp \left\{[f(L \bar{h}), \bar{h}]_{t}-\frac{1}{2}\|f(L \bar{h})\|_{t}^{2}-\frac{1}{2} \int_{0}^{t} f^{\prime}\left((L \bar{h})_{s}\right) \mathrm{d} s\right\}
$$

with $[\cdot, \cdot]_{t}$ and $\|\cdot\|_{t}$ denoting the inner product and norm in $\bar{H}^{t}$, respectively. Therefore,

$$
\begin{aligned}
& \int_{B} x_{t}\left(\bar{h}_{1}\right) B(t, x, y) \mathrm{d} \bar{\mu}\left(\bar{h}_{1}\right) \\
& =\int_{\bar{H}^{t}}(L \bar{h})_{t} B(t, L \bar{h}, y) B(t, f(L \bar{h}), \bar{h}) \\
& \quad \times \exp \left\{-\frac{1}{2} \int_{0}^{t} f^{\prime}\left((L \bar{h})_{s}\right) \mathrm{d} s\right\} \mathrm{d} \bar{\mu}^{t}(\bar{h}) .
\end{aligned}
$$

Using the Beners condition (38)-(39) and substituting in (40), gives us

$$
\begin{aligned}
& \int_{B} x_{t}\left(\bar{h}_{1}\right) B(t, x, y) \mathrm{d} \bar{\mu}\left(\bar{h}_{1}\right) \\
& =\mathrm{e}^{-c t / 2} \int_{B^{t}}(L \bar{h})_{t} \mathrm{e}^{[f(L \bar{h}), \bar{h}]_{t}} B\left(t,(1+a)^{1 / 2} L \bar{h}, \tilde{y}\right) \mathrm{d} \bar{\mu}^{t}(\bar{h}),
\end{aligned}
$$


where

$$
\tilde{y}_{t} \triangleq \frac{y_{t}-\frac{b}{2}}{(1+a)^{1 / 2}}
$$

and the condition $a>-1$ implies that $(1+a)^{1 / 2}$ is well-defined. Furthermore, by Theorem $4.5,[f(L \bar{h}), \bar{h}]_{t}$ is a p.r.v. and $f(\cdot)$ satisfying the Lipschitz and growth conditions above implies that

$$
\int_{H^{i}}(L \bar{h})_{t} \exp \left\{[f(L \bar{h}), \bar{h}]_{t}\right\} \mathrm{d} \bar{\mu}^{t}(\bar{h})
$$

is well defined. Hence we may express (35) as

$$
\psi_{t}(y)=\frac{R_{t}(\tilde{y})}{S_{t}(\tilde{y})}
$$

where

$$
R_{t}(z)=\frac{\int_{\mathscr{A}}(L \bar{h})_{t} \exp \left\{[f(L \bar{h}), \bar{h}]_{t}\right\} B\left(t,(1+a)^{1 / 2} L \bar{h}, z\right) \mathrm{d} \bar{\mu}(\bar{h})}{\int_{\tilde{H}} B\left(t,(1+a)^{1 / 2} L \bar{h}, z\right) \mathrm{d} \bar{\mu}(\bar{h})}
$$

and

$$
S_{t}(z)=\frac{\int_{\bar{H}} \exp \left\{[f(L \bar{h}), \bar{h}]_{t}\right\} B\left(t,(1+a)^{1 / 2} L \bar{h}, z \mathrm{~d} \bar{\mu}(\bar{h})\right.}{\int_{\bar{H}} B\left(t,(1+a)^{1 / 2} L \bar{h}, z \mathrm{~d} \bar{\mu}(\bar{h})\right.},
$$

Both (42) and (43) are in the form of the Kallianpur-Striebel formula. Thus (42) may be interpreted as the conditional expectation of the stochastic process $\bar{x}_{t}(\bar{h})=$ $(L \bar{h})_{t} \exp \left\{[f(L \bar{h}), \bar{h}]_{t}\right\}$ given the observation

$$
\bar{y}_{s}=(1+a)^{1 / 2}(L \bar{h})_{s}+\overline{\bar{h}}_{s}, \quad 0 \leqslant s \leqslant t,
$$

with $(\bar{h}, \overline{\bar{h}}) \in H$. The interpretation of (43) is similar. We can then write the nonlinear filter map as

$$
\psi_{t}(y)=\frac{r_{t}(K y, P)}{s_{t}(K y, P)},
$$

where $r_{t}(K y, P)$ corresponds to the expectation under the Gauss measure with mean $K y$ and covariance $P=(I-K)\left(I-K^{*}\right)$ of $(L \bar{h})_{t} \exp \left\{[f(L \bar{h}), \bar{h}]_{t}\right\}$ and, similarly, $s_{t}(K y, P)$ the expectation under the same Gauss measure of $\exp \left\{[f(L \bar{h}), \bar{h}]_{t}\right\}$. Here

$$
K=(1+a)^{1 / 2} L^{*}\left[I+(1+a) L L^{*}\right]^{-1} \text {. }
$$

We can interpret $K$ as resulting from a linear Gaussian problem. 


\section{Conclusion}

We gave a survey of some very recent results on the direct modelling of white noise using finitely additive measures on Hilbert spaces. The emphasis was on developing a framework to model the state equation also directly (along with the observation equation) with 'finitely additive' white noise driving term. The fact that the state process thus obtained is a Markov process opens up a lot of new possibilities. One of them is the study of robust nonlinear filtering for correlated state and observation processes. The other is calculating the Radon-Nikodym derivative of the derivative of the state process with respect to the canonical Gauss measure. This result, in turn, leads directly to the Wong-Zakai correction term and a representation result for nonlinear filter maps which closely resembles results on finite dimensional filters under Benès condition. Another important advantage of directly modelling the state process is to study the stochastic differential equations satisfying prescribed boundary conditions, an area which saw some advances recently [18]. We did not discuss them in this paper. It is also possible to study partial differential equations with white noise driving terms. We refer to the paper of Leland [16] for recent results in this area and the references.

\section{References}

1. Bagchi, A.: Cylindrical measures in Hilbert space and likelihood ratio for two-parameter signals in additive white noise, Control Theory Advanced Technol. 1 (1985), 139-153.

2. Bagchi, A. and Karandikar, R.: White noise theory of robust nonlinear filtering with correlated state and observation noises, Systems Control Lett. 1994 (to appear).

3. Bagchi, A. and Mazumdar, R.: On Radon-Nikodym derivatives of finitely additive measures induced by nonlinear transformations on Hilbert space, J. Nonlinear Anal. 21 (1993), 879-902.

4. Balakrishnan, A. V.: Applied Functional Analysis, 2nd edn, Springer-Verlag, New York, 1981.

5. Beněs, V. E.: Exact finite dimensional filters for certain diffusions with nonlinear drift, Stochastics 5 (1981), 65-82.

6. Berger, M. E.: Nonlinearity and Functional Analysis, Academic Press, New York, 1977.

7. Cameron, R. H. and Martin, M. T.: The transformation of Wiener integrals by nonlinear transformations, Trans. Amer. Math. Soc. 66 (1949), 253-283.

8. Dunford, N. and Schwartz, J. T.: Linear Operators, Vol. II, Interscience, New York, 1963.

9. Elliott, R. J.: Stochastic Calculus and Applications, Springer-Verlag, New York, 1982.

10. Fefferman, C.: A Radon-Nikodym theorem for finitely additive set functions, Pacific J. Math. 23 (1967), 35-45.

11. Gross, L.: Integration and nonlinear transformation in Hilbert space, Trans. Amer. Math. Soc. 94 (1960), 404-440.

12. Gross, L.: Abstract Wiener spaces, in Proceedings of the Fifth Berkeley Symposium on Probability and Statistics, Vol. II, Part I, pp. 31-42, 1965.

13. Ikeda, N. and Watanabe, S.: Stochastic Differential Equations and Diffusion Processes, NorthHolland, Amsterdam, 1986.

14. Kallianpur, G.: Stochastic Filtering Theory, Springer-Verlag, New York, 1980.

15. Kallianpur, G. and Karandikar, R. L.: White Noise Theory of Filtering, Prediction and Smoothing, Gordon and Breach, London, 1988.

16. Leland, R. P.: White noise in atmospheric optics, Acta Applic. Math. 35 (1994), 103-130.

17. Mazumdar, R. and Bagchi, A.: A representation result for nonlinear filter maps in a white noise framework, TW Memorandum No. 1062, University of Twente, The Netherlands (submitted for publication). 
18. Nualart, D. and Pardoux, E.: Boundary value problems for stochastic differential equations, Ann. Probab. 19 (1991), 1118-1144.

19. Prenter, P. M.: A Weierstrass theorem for real, separable Hilbert spaces, J. Approx. Theory 3 (1970), 341-351.

20. Prohorov, Yu. V.: Convergence of random processes and limit theorems of probability theory, Theory Probab. Appl. 1 (1956), 177-238.

21. Ramer, R.: On nonlinear transformation of Gaussian measures, J. Functional Anal. 15 (1974), 166-187.

22. de Santis, A., Gandolfi, A., Germany A. and Tardelli, P.: A representation theorem for a class of Radon-Nikodym derivatives in the white noise theory, Bull. Amer. Math. Soc. 1994 (to appear).

23. Wong, E. and Hajek, B.: Stochastic Processes in Engineering Systems, Springer-Verlag, New York, 1985.

24. Wong, E. and Zakai, M.: Riemann-Stieltjes approximations of stochastic integrals, Z. Wahrsch. verw. Geb. 12 (1969), 87-97. 